\title{
The effects of TRAF6 on proliferation, apoptosis and invasion in osteosarcoma are regulated by miR-124
}

\author{
QINGBING MENG ${ }^{*}$, WENSHENG ZHANG ${ }^{*}$, XINGLI XU, JIAN LI, HONGXIN MU, \\ XIAOLAN LIU, LING QIN, XIAOQI ZHU and MINQIAN ZHENG
}

\author{
Orthopedics Department, Yancheng City No. 1 People's Hospital, Yancheng, Jiangsu 224005, P.R. China
}

Received June 29, 2016; Accepted January 19, 2018

DOI: $10.3892 /$ ijmm.2018.3458

\begin{abstract}
The present study aimed to verify tumor necrosis factor receptor-associated factor 6 (TRAF6) as the target gene of microRNA-124 (miR-124). In addition, the expression of miR-124 was investigated in osteosarcoma tissues and cells, and its effects on the biological characteristics of osteosarcoma cells were determined, in order to provide an experimental and theoretical basis for the application of TRAF6 in the treatment of osteosarcoma. A fluorescence reporter enzyme system was used to verify TRAF6 as a target gene of miR-124, and western blotting was used to detect the effects of miR-124 on the protein expression levels of TRAF6 in cells. The expression levels of miR-124 were detected in osteosarcoma tissues and an osteosarcoma cell line (MG-63) by quantitative polymerase chain reaction (qPCR). In addition, a total of $48 \mathrm{~h}$ post-transfection of MG-63 cells with a miR-124 mimic, qPCR was used to detect the expression levels of miR-124, and the effects of miR-124 on the viability of MG-63 human osteosarcoma cells was determined using the MTT method. The effects of miR-124 on the cell cycle progression and apoptosis of MG-63 cells were analyzed by flow cytometry, whereas the effects of miR-124 on the migration of MG-63 cells was detected using the Transwell invasion chamber analysis method. A TRAF6 recombinant expression plasmid (pcDNA3.1-TRAF6) was also constructed, and MG-63 cells were transfected with the recombinant plasmid and a miR-124 mimic, in order to further validate the biological role of miR-124 via the regulation of TRAF6. The results of the present study indicated that, compared with in the normal control group, the expression levels of miR-124 were significantly increased in MG-63 cells transfected with a miR-124 mimic $(\mathrm{P}<0.01)$. In addition, the luciferase reporter gene system demonstrated that, compared with in the control group, relative
\end{abstract}

Correspondence to: Professor Minqian Zheng, Orthopedics Department, Yancheng City No. 1 People's Hospital, 16 Yue-He Road, Yancheng, Jiangsu 224005, P.R. China

E-mail: yczhengmq@163.com

*Contributed equally

Key words: osteosarcoma, tumor necrosis factor receptor-associated factor 6, microRNA-124, apoptosis, metastasis luciferase activity was significantly reduced in the miR-124 mimic group $(\mathrm{P}<0.01)$. The results of MTT analysis indicated that cell viability was also significantly reduced in response to the overexpression of miR-124 in MG-63 cells $(\mathrm{P}<0.01)$. Flow cytometric analysis demonstrated that the proportion of cells in $\mathrm{S}$ phase and $\mathrm{G}_{2} / \mathrm{M}$ phase was significantly decreased $(\mathrm{P}<0.01)$ in cells overexpressing miR-124, and the number of apoptotic cells was significantly increased $(\mathrm{P}<0.01)$. Furthermore, the results of the Transwell invasion assay suggested that the number of invasive cells was significantly decreased following enhanced expression of miR-124 ( $\mathrm{P}<0.01)$. In MG-63 cells overexpressing miR-124 and TRAF6, the results of MTT, flow cytometric and Transwell assay analyses demonstrated that the overexpression of TRAF6 had the opposite biological effects compared to miR-124 overexpression. In conclusion, the present study indicated that the expression levels of miR-124 were downregulated in human osteosarcoma tissues and cells, and that miR-124 is associated with negative regulation of TRAF6 expression; therefore, the role of TRAF6 in primary osteosarcoma may be regulated by miR-124. Therapeutic strategies that enhance miR-124 expression or inhibit TRAF6 expression may be beneficial for the treatment of patients with osteosarcoma.

\section{Introduction}

Osteosarcoma, which is also known as osteogenic sarcoma, is a malignant form of bone tumor that more commonly occurs in adolescents and children <20 years old (1); osteosarcoma accounts for $\sim 5 \%$ of pediatric tumors (2). Osteosarcoma is associated with high malignancy and rapid progress, and can induce early lung metastasis. Furthermore, osteosarcoma has a high recurrence rate and poor prognosis; the 5-year survival rate of patients with osteosarcoma is only $20 \%$, and it is associated with a heavy burden to family members and society (3-5). In recent years, with the development of neoadjuvant chemotherapy/postoperative adjuvant chemotherapy, and the combination of surgical treatment and radiotherapy, the prognosis of patients with osteosarcoma has improved; however, the 5-year survival rate could only be increased to $\sim 60 \%(6-8)$. Numerous studies have been conducted by researchers with regards to the mechanisms underlying the occurrence and development of osteosarcoma; however, the pathogenesis of osteosarcoma remains to be elucidated. Therefore, the pathogenesis of osteosarcoma requires further 
study, in order to provide scientific evidence for the diagnosis, treatment and prognosis of osteosarcoma. Elucidation of the pathogenesis of osteosarcoma may result in more effective control of the pathogenic process of osteosarcoma, and may improve patient prognosis, and reduce the burden on patients, families and society $(9,10)$.

Tumor occurrence and development are the results of numerous gene mutations, including activation of oncogenes and inactivation of tumor suppressor genes $(11,12)$. At present, the majority of studies have suggested that activation of oncogenes serves an important role in tumor occurrence and development $(13,14)$. During the occurrence and development of tumors, tumor necrosis factor receptor-associated factor 6 (TRAF6) functions as a cancer gene $(15,16)$. TRAF6 belongs to the TRAF family; TRAF is a cytoplasmic adapter protein, which comprises a family of seven members (TRAF 1-7). These members serve important roles in various signaling pathways, including the tumor necrosis factor receptor superfamily and interleukin-1 receptor superfamily, which are involved in regulating cell survival and cell death (17-19). TRAF6 is closely associated with the maturation and differentiation of osteoclasts in vitro (20). Recent studies have reported that TRAF6 serves an important role in the development, invasion and metastasis of tumors by activating nuclear factor $(\mathrm{NF})-\kappa \mathrm{B}(21,22)$. However, studies regarding the expression of TRAF6 in osteosarcoma and its biological role are rare.

Previous studies have revealed that TRAF6 is highly expressed in lung cancer, glioma and esophageal cancer, and it is associated with tumor invasion and metastasis (15,23-25). In addition, it has been suggested that TRAF6 may promote tumor angiogenesis (26). Our previous study indicated that TRAF6 mRNA and protein was highly expressed in osteosarcoma tissues (27). This finding was consistent with the expression pattern of TRAF6 in other tumors. Numerous studies have been conducted in the field of osteosarcoma; however, studies regarding the mechanism underlying the effects of TRAF6 on the pathogenesis of osteosarcoma are rare, and why TRAF6 expression is upregulated in osteosarcoma remains unclear. Therefore, research regarding the regulation of TRAF6 expression may have important theoretical significance and practical value.

In recent years, a novel method of gene expression regulation, namely microRNA (miRNA/miR) regulation, has been discovered $(28,29)$. The present study aimed to determine whether the high expression of TRAF6 in osteosarcoma cells was due to miRNA regulation. Therefore, databases, including TargetScan, miRanda and PicTar, were analyzed using online software, so as to predict the possible regulatory effects of miRNA on TRAF6, thus resulting in the identification of miR-124. It has previously been reported that miR-124 exhibits low expression in breast cancer tissues, whereas overexpression of miR-124 could inhibit the proliferation and migratory ability of breast cancer cells (30). In addition, overexpression of miR-124 was able to inhibit the invasion and migration of ovarian cancer cells (31). Therefore, the present study hypothesized that the biological effects of TRAF6 in osteosarcoma were regulated by miR-124. The present study confirmed that TRAF6 was a target gene of miR-124 using a luciferase reporter gene system, after which the biological effects of miR-124 on human osteosarcoma cells were determined according to a series of experiments. The present study further tested and verified the biological role of miR-124 via the regulation of TRAF6 using an overexpression vector.

\section{Materials and methods}

Specimens. A total of 16 fresh osteosarcoma specimens and normal bone tissues were collected from patients with osteosarcoma ( $n=16 ; 8$ male and 7 female; 11 younger than 40 years old and 5 older than 40 years old) by surgical excision; specimens were immediately stored in liquid nitrogen. None of the patients had a history of chemotherapy, radiotherapy or other treatments, and did not have other inflammatory diseases. The present study was approved by the Ethics Committee of Yancheng City No. 1 People's Hospital (Yancheng, China; approval no. 2015YCYY-0001). The patients provided written informed consent prior to sample collection.

Main reagents. The miR-124 reverse transcription ( $\mathrm{RT}$ ) primer (5'-GATACTCATAAGGCACGCGG-3' and 5'-GTGCAG GGTCCGAGGT-3'), miR-124 mimic and non-specific control (mimic control) were purchased from Shanghai GenePharma Co., Ltd. (Shanghai, China).

The TaqMan miRNA isolation kit, TaqMan miRNA assay and TaqMan Universal polymerase chain reaction (PCR) master mix were purchased from Applied Biosystems; Thermo Fisher Scientific, Inc. (Waltham, MA, USA).

Fetal bovine serum (FBS), Dulbecco's modified Eagle's medium (DMEM), Eagle's Minimum Essential medium (EMEM), L-glutamine and HEPES were purchased from Gibco; Thermo Fisher Scientific, Inc., and Lipofectamine ${ }^{\circledR} 2000$ was purchased from Invitrogen; Thermo Fisher Scientific, Inc. Nunc $^{\mathrm{TM}}$ cell culture plates and culture dishes were obtained from Thermo Fisher Scientific, Inc., and the Transwell invasion chamber was purchased from Corning Incorporated (Corning, NY, USA).

MTT and radioimmunoprecipitation assay (RIPA) lysis buffer were purchased from Beyotime Institute of Biotechnology (Shanghai, China). Trypsin, PBS and Hoechst 33342 were purchased from Sigma-Aldrich; Merck KGaA (Darmstadt, Germany). Matrigel was purchased from BD Biosciences (Franklin Lakes, NJ, USA). Annexin V and propidium iodide (PI) were purchased from Roche Diagnostics (Indianapolis, IN, USA). Rabbit anti-human TRAF6 polyclonal antibody and mouse anti-human $\beta$-actin monoclonal antibody were purchased from Abcam (Cambridge, MA, USA). Horseradish peroxidase (HRP)-conjugated affinity-purified goat anti-mouse and goat anti-rabbit immunoglobulin (Ig)G secondary antibodies were purchased from Sigma-Aldrich; Merck KgaA. A protein extraction and quantification kit (BCA kit) was purchased from Bio-Rad Laboratories, Inc. (Hercules, CA, USA).

The carrier pcDNA3.1, pGEM-T and pGL3-Basic vectors, competent cells (DH5 $\alpha$ ) and TRIzol ${ }^{\circledR}$ total RNA extraction kit were purchased from Invitrogen; Thermo Fisher Scientific, Inc. BamHI and XhoI restriction enzymes, and DNA Marker were purchased from Takara Bio, Inc. (Otsu, Japan). T4 DNA ligase was purchased from Promega Corporation (Madison, WI, USA). Taq DNA polymerase and prestained protein marker were purchased from Fermentas; Thermo Fisher Scientific, Inc. Transcriptor First Strand cDNA synthesis kit was purchased from Qiagen GmbH (Hilden, Germany). 
Cell treatment. MG-63 cells were purchased from Shanghai Cell Bank of Chinese Academy of Sciences (Shanghai, China), and were cultured in EMEM (2 mM L-glutamine, $1.0 \mathrm{mM}$ sodium pyruvate, $0.1 \mathrm{mM}$ non-essential amino-acid) containing 10\% FBS. 293 cells were purchased from American Type Culture Collection (Manassas, VA, USA), and were cultured in DMEM containing 10\% FBS.

Construction of recombinant TRAF6 expression plasmids. According to the human TRAF6 mRNA sequence in GenBank (GI: 1732425; https://www.ncbi.nlm.nih.gov/genbank/) and enzyme cleavage site analysis, a pair of primers was designed using Primer Premier 5 software (Premier Biosoft International, Palo Alto, CA, USA) in the two flanks of the open reading frame of TRAF6. The primer sequences were as follows: Upstream primer P1, 5'-CGGGATCCATGAGTCT GCTAAACTGTGA-3', which contained the BamHI enzyme cleavage site; and downstream primer P2, 5'-CCCTCGAGTAC CCCTGCATCAGTACTTC-3', which contained the XhoI enzyme cleavage site (the underlined sections of the primer sequences indicate the restriction endonuclease site). The primers were synthesized by Sangon Biotech Co., Ltd. (Shanghai, China).

Cultured MG-63 cells were collected and treated with $1 \mathrm{ml}$ TRIzol ${ }^{\circledR}$ reagent; total RNA was extracted according to manufacturer's protocol. RT was conducted using a first strand cDNA synthesis kit, and the first chain was produced according to the manufacturer's protocol. The TRAF6 primer was dissolved in $\mathrm{ddH}_{2} \mathrm{O}$ and used to amplify TRAF6 by PCR. The PCR product was then ligated into the pGEM-T carrier under T4 DNA ligase, so as to screen positive clones. The positive clone was verified by sequencing, and the subclone was merged into the pcDNA3.1 expression vector to construct the recombinant expression vector, pcDNA3.1-TRAF6. The RT thermocycling conditions were as follows: $16^{\circ} \mathrm{C}$ for $30 \mathrm{~min}, 42^{\circ} \mathrm{C}$ for $30 \mathrm{~min}, 85^{\circ} \mathrm{C}$ for $5 \mathrm{~min}$; in the end hold at $4^{\circ} \mathrm{C}$. The PCR conditions were as follows: $95^{\circ} \mathrm{C}$ for $10 \mathrm{~min}, 95^{\circ} \mathrm{C}$ for $10 \mathrm{sec}, 60^{\circ} \mathrm{C}$ for $60 \mathrm{sec}(40$ cycles).

Cell transfection. Cultured MG-63 cells were uniformly inoculated into 6-well culture plates at $3 \times 10^{5} / \mathrm{ml}$; the volume of each well was $1 \mathrm{ml}$. Following growth of adherent cells, transfection with miR-124 mimic, mimic control, pcDNA3.1-TRAF6 recombinant plasmid or empty plasmid control (pcDNA3.1) was conducted using Lipofectamine ${ }^{\circledR} 2000$ according to the manufacturer's protocol. In addition, a normal control group (normal; untransfected cells served as the normal control) was generated. Briefly, miR-124 mimic, mimic control, pcDNA3.1-TRAF6 and pcDNA3.1 were diluted in minimum essential medium (MEM; Thermo Fisher Scientific, Inc.). Subsequently, Lipofectamine ${ }^{\circledR} 2000$ was diluted in MEM, and was incubated for $5 \mathrm{~min}$ at room temperature after gentle agitation. The diluted Lipofectamine ${ }^{\circledR} 2000$ was mixed with the diluted miR-124 mimic or mimic control, and was incubated for $20 \mathrm{~min}$ at room temperature after gentle agitation, so as to form a compound. The compound was then added to the culture plate containing MG-63 cells, and was incubated at $37^{\circ} \mathrm{C}$ in an atmosphere containing $5 \% \mathrm{CO}_{2}$. After $5 \mathrm{~h}$, the medium was replaced with MEM containing 10\% FBS for a further $48 \mathrm{~h}$.

Luciferase reporter gene system. The analysis result of TargetScan (http://www.targetscan.org) demonstrated that TRAF6 was one of target gene of miR-124. Luciferase plasmids were constructed, as follows: A sequence containing a fragment of the wild-type 3'-untranslated region (3'-UTR) of the target gene, TRAF6, was chemically synthesized, which was complementary to the corresponding miR-124. Subsequently, the sequence fragment of the TRAF6 3'-UTR (wild-type) containing the predicted miR-124 binding sites, and a mutated sequence fragment of the TRAF6 3'-UTR (mutant) were cloned into the $\mathrm{XbaI}$ site of a pGL3-Basic carrier. The PCR primer with mutant site was synthesized and mutant 3'-UTR was generated by PCR amplification.

Cultured 293 cells were uniformly inoculated into 6-well culture plates at $3 \times 10^{5} / \mathrm{ml}$; the volume of each well was $1 \mathrm{ml}$. Subsequently, the cells were transfected with the luciferase plasmids containing TRAF6 3'-UTR (wild-type) or mutated TRAF6 3'-UTR (mutant), alongside the miR-124 mimic or mimic control. In addition, a normal control group was generated. A total of $48 \mathrm{~h}$ post-transfection, cells were collected and detected using a luciferase kit; a microplate reader was used for numerical readings at a wavelength of $560 \mathrm{~nm}$.

Western blot analysis. Proteins were extracted from MG-63 cells using RIPA lysis buffer post-transfection with a miR-124 mimic or mimic control for $48 \mathrm{~h}$. The concentration in the supernatant was then measured using the bicinchoninic acid method. Proteins $(50 \mu \mathrm{g})$ were separated by $5-15 \%$ SDS-PAGE and were transferred onto a polyvinylidene fluoride membrane via Wet Transfer (Bio-Rad Laboratories, Inc.). The membrane was blocked for $1 \mathrm{~h}$ at room temperature in TBST $(10 \mathrm{mM}$ Tris-HCl, pH 7.5, $150 \mathrm{mM} \mathrm{NaCl}$ and $0.1 \%$ Tween-20) containing $5 \%$ skimmed milk powder. Subsequently, the membrane was incubated with rabbit anti-human TRAF6 polyclonal antibody (1:500 dilution; ab181622; Abcam) and rabbit anti-human $\beta$-actin monoclonal antibody (1:1,000 dilution; ab8227; Abcam) overnight at $4^{\circ} \mathrm{C}$, and was washed three times with TBST (5 min/wash). The membrane was then incubated with HRP-conjugated goat anti-rabbit (A6154; Sigma-Aldrich; Merck $\mathrm{KgaA}$ ) or goat anti-mouse (A4416; Sigma-Aldrich; Merck KgaA) IgG secondary antibodies (dilution, $1: 2,000$ ) at $37^{\circ} \mathrm{C}$ for $1 \mathrm{~h}$, after which it was washed three times with TBST ( $5 \mathrm{~min} /$ wash). Blots were visualized using an enhanced chemiluminescence reagent (Thermo Fisher Scientific, Inc.). Relative protein expression levels of TRAF6 were expressed as the gray value ratio of TRAF6/ $\beta$-actin; relative expression alterations were analyzed by PDQuest software (version 8.0; Bio-Rad Laboratories, Inc.).

MTT assay. After MG-63 cells were transfected with a miR-124 mimic or mimic control for $48 \mathrm{~h}$, RNA was extracted from the cells in each group using the TaqMan miRNA isolation kit. Alterations in the expression levels of miR-124 were detected in each group using reverse transcription-quantitative PCR (RT-qPCR).

After MG-63 cells were transfected with a miR-124 mimic or mimic control for $48 \mathrm{~h}$, they were incubated with $100 \mu \mathrm{l}$ $\operatorname{MTT}(0.5 \mathrm{mg} / \mathrm{ml})$ solution/well at $37^{\circ} \mathrm{C}$ in an atmosphere containing $5 \% \mathrm{CO}_{2}$ for $4 \mathrm{~h}$. Subsequently, $100 \mu \mathrm{l} 20 \%$ SDS (auxiliary solvent, 50\% dimethylfomamide) was added to each well for $24 \mathrm{~h}$ at $37^{\circ} \mathrm{C}$. A microplate reader (Bio-Tek Instruments, Inc., Winooski, VT, USA) was used to detect the optical density values at $570 \mathrm{~nm}$. In addition, the normal control group was analyzed. A total of 10 complex holes were 
set up in each experimental group, and the experiment was repeated three times.

Flow cytometric detection of cell cycle progression. MG-63 cells were transfected with a miR-124 mimic or mimic control for $48 \mathrm{~h}$. In addition, a normal control group (normal) was generated. Subsequently, the cells were washed 1-2 times with PBS and trypsin was used to digest cells, after which cells were washed a further 1-2 times with PBS and the cells were collected by centrifugation. PI was added to the cells, which were incubated in the dark for $30 \mathrm{~min}$ at $4^{\circ} \mathrm{C}$. Finally, cells were filtered and underwent flow cytometric analysis (BD Biosciences). CellQuest software (BD Biosciences) was used to count cells, and FACsuite software (BD Biosciences) was used to analyze the data.

Flow cytometric analysis of apoptosis. MG-63 cells were transfected with a miR-124 mimic or mimic control for $48 \mathrm{~h}$. In addition, a normal control group (normal) was generated. Subsequently, the cells were washed 1-2 times with PBS, and were incubated with Annexin V-fluorescein isothiocyanate and PI in the dark for $15 \mathrm{~min}$ at room temperature. Finally cells were filtered and underwent flow cytometric analysis (BD Biosciences) for the detection of apoptotic cells. CellQuest software (BD Biosciences) was used to count cells, and Mac quit software was used to analyze the data.

Transwell invasion chamber assay. Transfection of MG-63 cells with a miR-124 mimic and mimic control was conducted using Lipofectamine ${ }^{\circledR} 2000$ according to the manufacturer's protocol. In addition, a normal control group (normal) was generated. MG-63 cells were transfected with the specified mimics for $24 \mathrm{~h}$; after transfection, the cells were seeded in a Transwell invasion chamber and incubated for another $24 \mathrm{~h}$, and after cultivation under normal conditions for $24 \mathrm{~h}$, the cells were washed 1-2 times with PBS, and the cells were stained with Hoechst 33342. Eight visual fields were observed at random, and the number of cells that passed through the Transwell polycarbonate membrane was counted under a microscope (Leica DM IL; Leica, Wetzlar, Germany). Cells that passed through the Transwell polycarbonate membrane were considered invasive cells. The Transwell chamber wells were coated in Matrigel.

Further validation of the role of TRAF6 in cell biology via miR-124 regulation. In order to confirm that TRAF6 serves a role in cell biology via miR-124 regulation, a TRAF6 recombinant expression vector, pcDNA3.1-TRAF6, was constructed. The cells were divided into the following five groups: Normal control group (normal), empty vector control group (pcDNA3.1), pcDNA3.1-TRAF6 transfection group and miR-124 mimic + pcDNA3.1-TRAF6 transfection group. The miR-124 mimic and pcDNA3.1-TRAF6 were simultaneously transfected into MG-63 cells using Lipofectamine ${ }^{\circledR} 2000$. For MTT assay, cultured MG-63 cells were uniformly inoculated into 96 -well culture plates at $3 \times 10^{5} / \mathrm{ml}$; the volume of each well was $0.1 \mathrm{ml}$. A total of $300 \mathrm{ng}$ pcDNA3.1-TRAF6 and $50 \mathrm{nM}$ mimic were then diluted into $25 \mu \mathrm{l}$ medium without serum. And $1 \mu 1$ Lipofectamine ${ }^{\circledR} 2000$ was diluted into $25 \mu 1$ OptiMEM Medium and incubate for $5 \mathrm{~min}$ at room temperature. The DNA- mimic-Lipofectamine ${ }^{\circledR} 2000$ complexes $(50 \mu \mathrm{l})$ were directly added to each well of the plates containing cells and
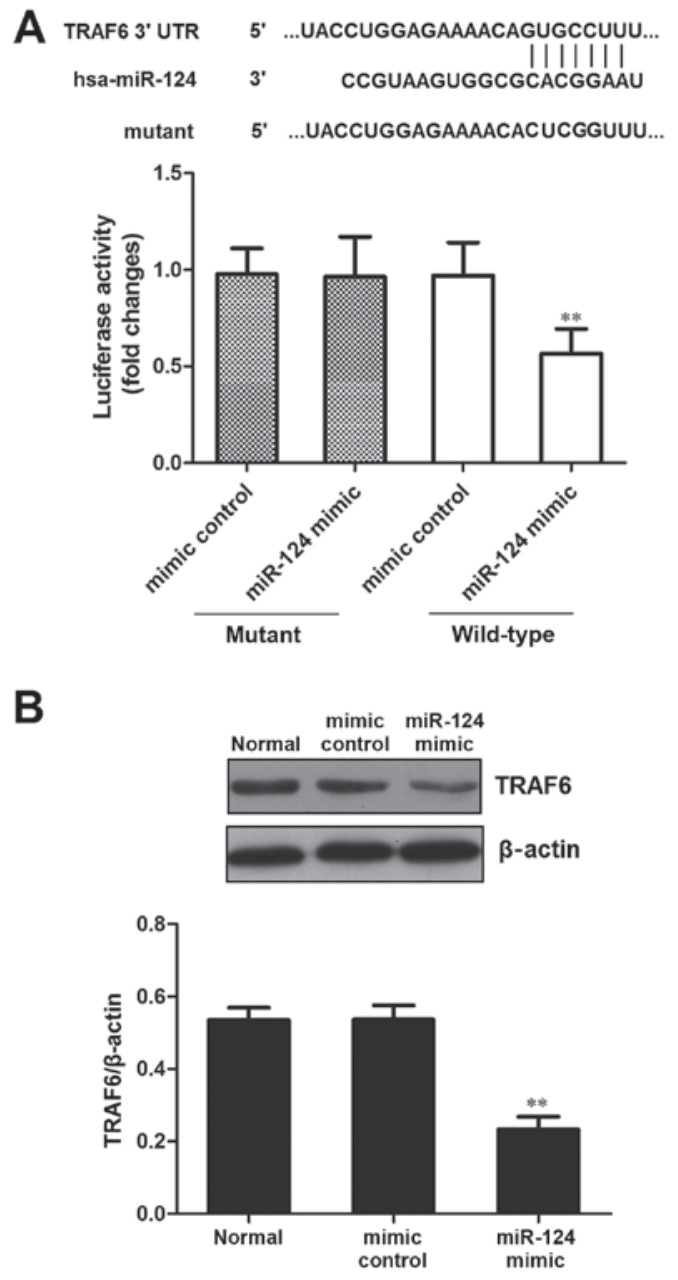

Figure 1. Luciferase reporter gene analysis was used to determine whether TRAF6 is a target gene of miR-124. (A) Upper panel, schematic representation of the TRAF6 3'-UTR showing the putative miR-124 binding site. Lower panel, statistical analysis of relative luciferase activity in each group. (B) Regulatory effects of miR-124 on TRAF6 expression, as detected by western blotting. ${ }^{* *} \mathrm{P}<0.01$ vs. the mimic control and normal groups. 3 '-UTR, 3'-untranslated region; miR-124, microRNA-124; TRAF6, tumor necrosis factor receptor-associated factor 6 .

were then mixed gently. To further validate the role of TRAF6 in cell biology via miR-124 regulation, MTT, flow cytometry and Transwell analytical methods were used.

Statistical analysis. The data are presented as mean \pm SD. The experiments were repeated 3 times. Statistical processing of experimental data was conducted using SPSS 17.0 statistical analysis software (SPSS, Inc., Chicago, IL, USA), and it was used to compare the differences between two groups. One-way analysis of variance was used to analyze the data and Tukey's post hoc test was used to analyze the differences between more than two groups. Student's t-test was used to analyze the differences between two groups. $\mathrm{P}<0.05$ was considered to indicate a statistically significant difference.

\section{Results}

Identification of TRAF6 as a target gene of miR-124. In order to verify that the predicted miR-124 binding site was located in the TRAF6 3'-UTR, luciferase plasmids containing TRAF6 


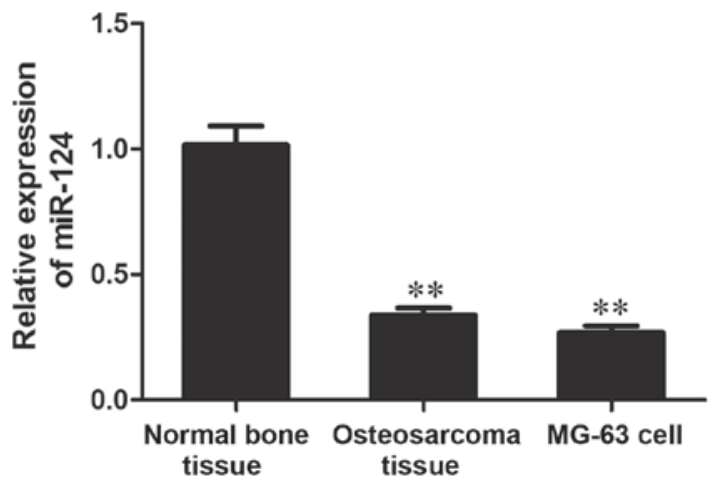

Figure 2. miR-124 expression in osteosarcoma tissues and MG-63 cells. ${ }^{* *} \mathrm{P}<0.01$ vs. normal bone tissue. miR-12, microRNA-124.

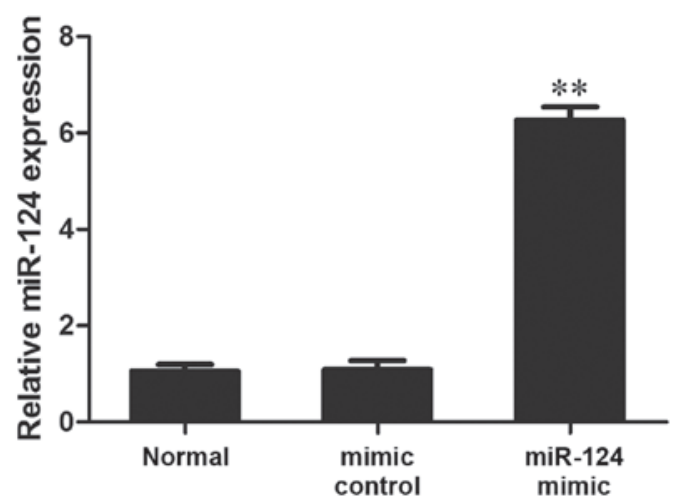

Figure 3. Effects of miR-124 mimic on miR-124 expression in MG-63 cells, as determined by reverse transcription-quantitative polymerase chain reaction. ${ }^{* *} \mathrm{P}<0.01$ vs. the normal or mimic control groups. miR-124, microRNA-124.

3'-UTR (wild-type) and mutated TRAF6 3'-UTR (mutant) were constructed, and were transfected into 293 cells alongside a miR-124 mimic or mimic control. The results demonstrated that compared with in the control mimic group, luciferase activity was significantly decreased in the miR-124 mimic and TRAF6 3'-UTR (wild-type) group ( $\mathrm{P}<0.01$; Fig. 1A). These results indicated that miR-124 may directly act on the predicted target site of TRAF6 3'-UTR.

Western blot analysis was used to detect TRAF6 expression and the effects of miR-124 on its regulation. The results indicated that the protein expression levels of TRAF6 were significantly reduced in the miR-124 mimic group compared with in the normal and mimic control groups $(\mathrm{P}<0.01$; Fig, 1B). These results suggested that overexpression of miR-124 inhibited the protein expression levels of TRAF6 protein.

miR-124 expression in human osteosarcoma tissues and cell lines. The results of RT-qPCR analysis demonstrated that the expression levels of miR-124 were significantly reduced in osteosarcoma tissues and MG-63 cells compared with in normal bone tissue $(\mathrm{P}<0.01$; Fig. 2$)$. These results suggested that miR-124 expression may be decreased in human osteosarcoma tissues and cell lines.

Effects of miR-124 on cell viability, as detected by MTT assay. Transfection of MG-63 cells with a miR-124 mimic induced

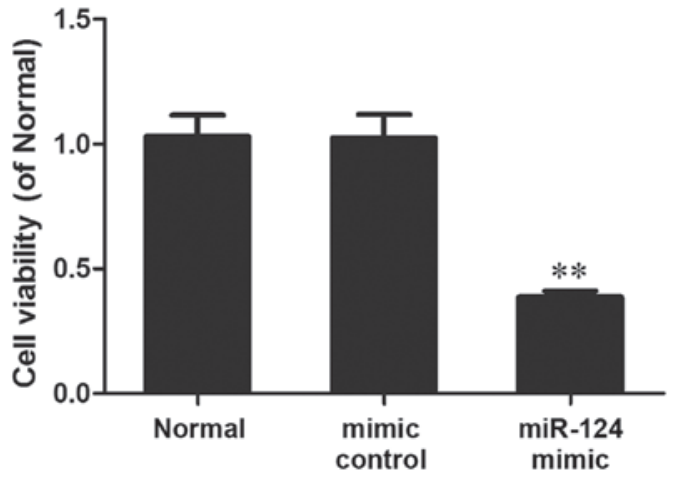

Figure 4. Effects of miR-124 overexpression on the viability of MG-63 cells, as determined by MTT analysis. ${ }^{* *} \mathrm{P}<0.01$ vs. the normal or mimic control groups. miR-124, microRNA-124.

miR-124 overexpression. The results of RT-qPCR analysis demonstrated that in the miR-124 mimic group, the expression levels of miR-124 were significantly increased compared with in the normal and mimic control groups $(\mathrm{P}<0.01$; Fig. 3$)$.

The results of the MTT analysis demonstrated that following transfection with a miR-124 mimic for $48 \mathrm{~h}$, cell viability was significantly reduced compared with in the normal and mimic control groups $(\mathrm{P}<0.01$; Fig. 4). These results indicated that miR-124 overexpression may reduce the viability of MG-63 cells.

Effects of miR-124 on cell cycle progression and apoptosis, as detected by flow cytometry. After MG-63 cells were transfected with a miR-124 mimic and mimic control for $48 \mathrm{~h}$, flow cytometry was used to detect the proportion of cells in each phase of the cell cycle. The results indicated that in the miR-124 mimic group, the proportion of $S$ phase cells and $\mathrm{G}_{2} / \mathrm{M}$ phase cells was significantly reduced compared with in the normal and mimic control groups $(\mathrm{P}<0.05)$, whereas the proportion of $\mathrm{G}_{0} / \mathrm{G}_{1}$ phase cells was significantly increased compared with in the normal and mimic control groups $(\mathrm{P}<0.05 ;$ Fig. $5 \mathrm{~A})$. These results indicated that miR-124 overexpression may inhibit cell cycle progression.

Flow cytometry was also used to determine the effects of miR-124 on cell apoptosis. The results demonstrated that in the miR-124 mimic group, the proportion of apoptotic cells was significantly higher compared with in the normal and mimic control groups $(\mathrm{P}<0.01$; Fig. 5B). These results suggested that miR-124 overexpression may promote cell apoptosis.

Effects of miR-124 on cell invasion, as detected by Transwell invasion assay. After MG-63 cells were transfected with a miR-124 mimic and mimic control for $48 \mathrm{~h}$, the number of cells that passed through the polycarbonate membrane of a Transwell invasion chamber was determined. Hoechst 33342 staining demonstrated that in the miR-124 mimic group, the number of cells that passed through the Transwell membrane was significantly decreased $(\mathrm{P}<0.01)$. In addition, the migration of MG-63 cells was inhibited post-transfection with a miR-124 mimic (Fig. 5C).

Validation of the role of miR-124 in cell biology via TRAF6 regulation. Our preliminary study confirmed that TRAF6 
A
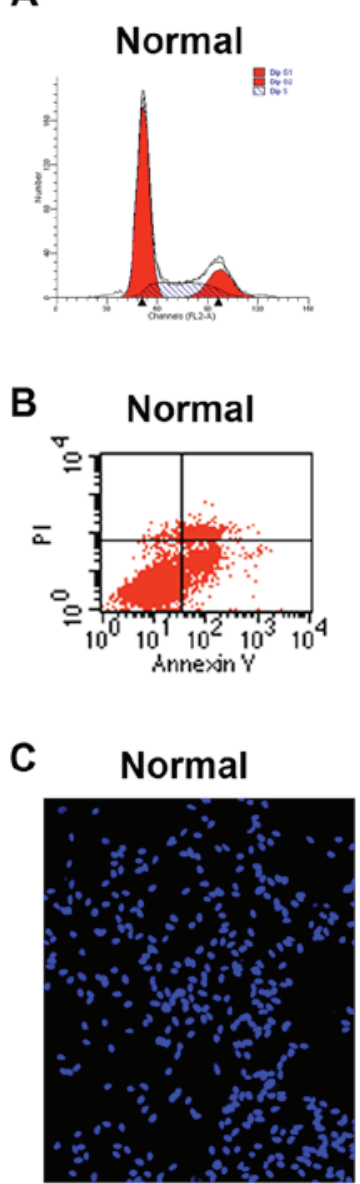

mimic control

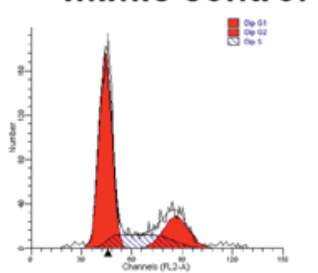

mimic control

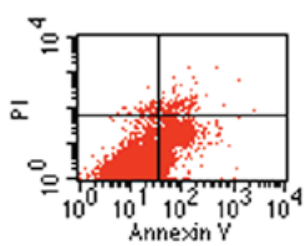

mimic control

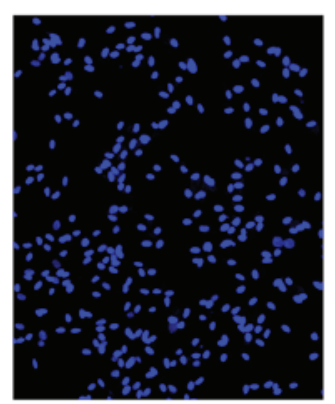

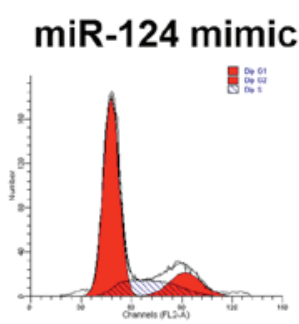

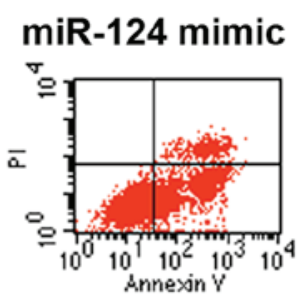

miR-124 mimic

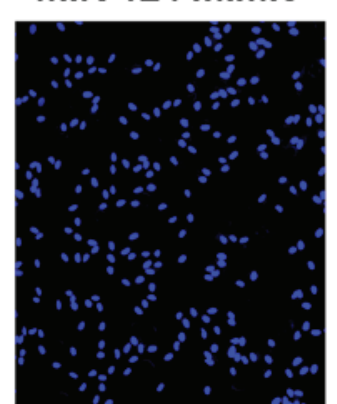

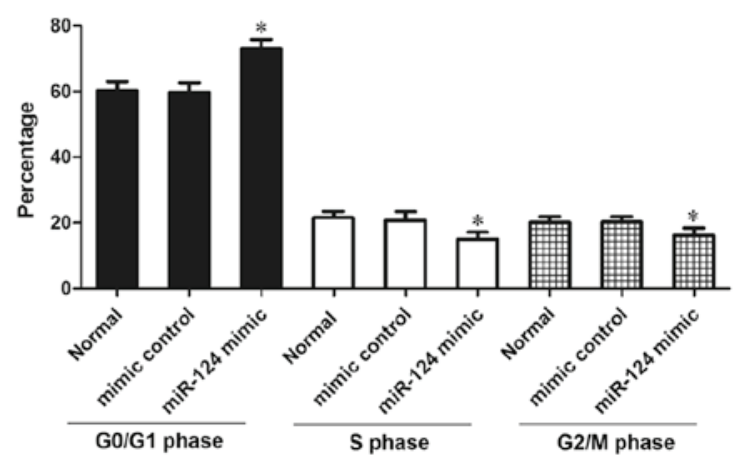
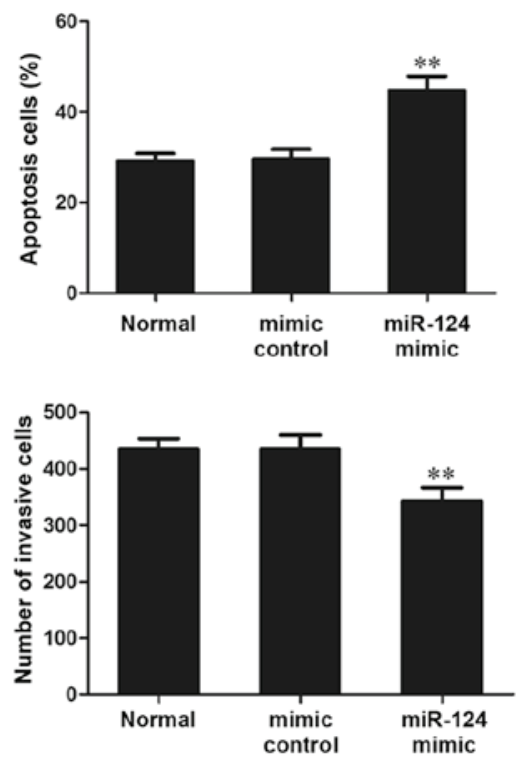

Figure 5. Effects of miR-124 on cell cycle progression, apoptosis and invasion. (A) Effects of miR-124 on cell cycle progression, as determined by flow cytometry. (B) Effects of miR-124 on cell apoptosis, as determined by flow cytometry. (C) Effects of miR-124 on cell invasion, as determined by Transwell invasion assay (magnification, $\mathrm{x} 200$ ). ${ }^{*} \mathrm{P}<0.05$ and ${ }^{* *} \mathrm{P}<0.01$ vs. the normal or mimic control groups. miR-124, microRNA-124; PI, propidium iodide.

served a role as a proto-oncogene in osteosarcoma (27). In addition, the results of the present study indicated that miR-124 may be considered a tumor suppressor gene in osteosarcoma, and TRAF6 was confirmed as a target gene regulated by miR-124. Therefore, the present study aimed to further validate the role of miR-124 in cell biology via TRAF6 regulation. Therefore, a recombinant expression plasmid of TRAF6 was constructed, after which MG-63 cells were cotransfected with a miR-124 mimic and the pcDNA3.1-TRAF6 recombinant expression vector using Lipofectamine ${ }^{\circledR} 2000$. Subsequently, the cells were evaluated using MTT, flow cytometry and Transwell analytical methods (Fig. 6). There were no significant differences between the miR-124 + pcDNA3.1-TRAF6 group and the pcDNA3.1-TRAF6 group.

Firstly, western blot analysis was used to detect the protein expression levels of TRAF6 in each group. The results revealed that transfection of MG-63 cells with pcDNA3.1-TRAF6 significantly promoted TRAF6 expression $(\mathrm{P}<0.01)$. However, in the miR-124 mimic + pcDNA3.1-TRAF6 transfection group, TRAF6 expression was significantly reduced compared with in the pcDNA3.1-TRAF6 transfection group $(\mathrm{P}<0.01)$, and was slightly higher than that in the normal and pcDNA3.1 groups ( $\mathrm{P}>0.05$; Fig. $6 \mathrm{~A})$.
Cell viability was increased in the pcDNA3.1-TRAF6 transfection group, and the proportion of cells in the $S+G_{2} / M$ phase was increased. Furthermore, the number of cells that passed through the polycarbonate membrane was significantly higher compared with in the other groups $(\mathrm{P}<0.01)$. These results suggested that TRAF6 may be considered a proto-oncogene in osteosarcoma. Cell viability in the miR-124 mimic + pcDNA3.1-TRAF6 transfection group was decreased, and the numbers of cells in $\mathrm{S}+\mathrm{G}_{2} / \mathrm{M}$ phase and that passed through the polycarbonate membrane were significantly reduced compared with in the pcDNA3.1-TRAF6 transfection group $(\mathrm{P}<0.01)$. Viability, cell cycle progression and invasion was slightly higher in the miR-124 mimic + pcDNA3.1-TRAF6 transfection group than in the normal and pcDNA3.1 groups (P>0.05; Fig. 6B, C and E). Furthermore, apoptosis was analyzed by flow cytometry, and the results revealed that in the miR-124 mimic + pcDNA3.1-TRAF6 transfection group, the number of apoptotic cells was significantly higher than in the pcDNA3.1-TRAF6 transfection group, and was slightly lower than in the normal and pcDNA3.1 groups ( $P>0.05$; Fig. 6D). These results indicated that miR-124 overexpression could inhibit TRAF6-induced cell cycle progression of osteosarcoma cells and initiate apoptosis. 
A

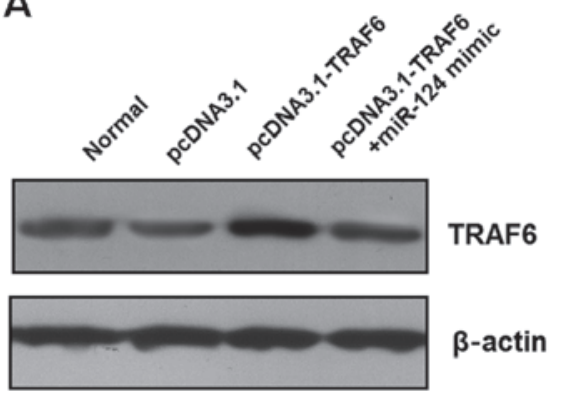

C

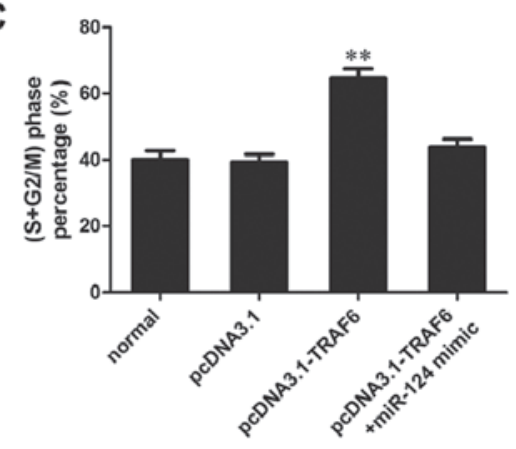

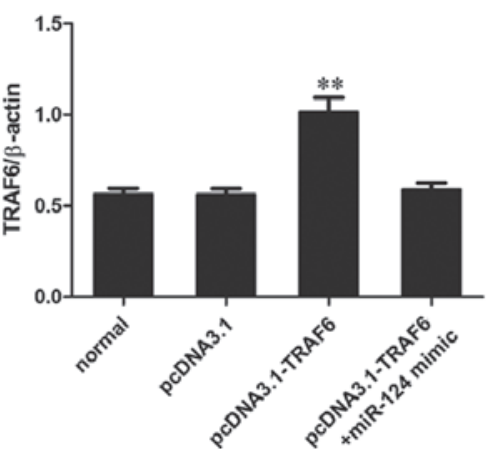

B
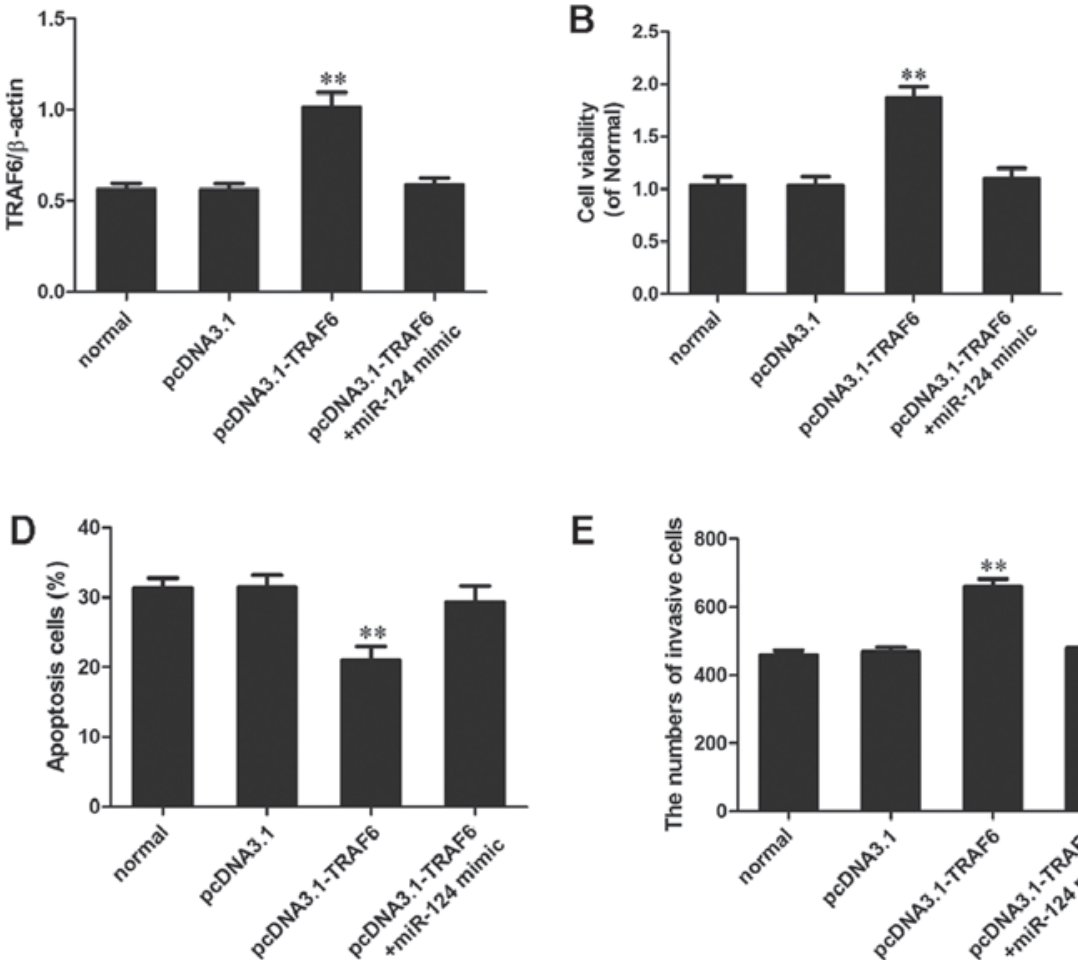

E

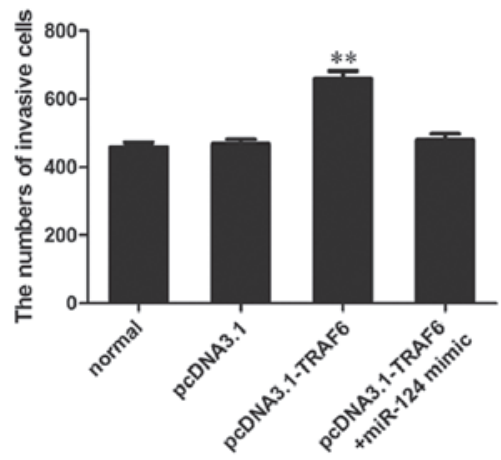

Figure 6. Rescue experiment was performed to verify the role of miR-124 in cell biology via the regulation of TRAF6. (A) TRAF6 expression was determined by western blotting in each group. (B) Viability of MG-63 cells, as detected by MTT analysis. (C) Cell cycle progression of MG-63 cells, as detected by flow cytometry. (D) Apoptosis of MG-63 cells, as detected by flow cytometry. (E) Invasion of MG-63 cells, as detected by Transwell invasion assay. ${ }^{* *}$ P $<0.01$ vs. the normal and pcDNA3.1 groups. miR-124, microRNA-214; TRAF6, tumor necrosis factor receptor-associated factor 6.

\section{Discussion}

Osteosarcoma often occurs in children and adolescents, and is one of the most common forms of primary malignant bone tumor $(32,33)$. Osteosarcoma is associated with a high degree of malignancy and early blood metastasis; in addition, the incidence of pulmonary metastasis is high, disease development is rapid and it is associated with high levels of mortality $(34,35)$. Increased information regarding the molecular mechanism underlying the occurrence and metastasis of osteosarcoma is required to serve a guiding role in gene-targeted therapy $(36,37)$.

During tumor occurrence and development, TRAF6 has a functional role as a cancer gene $(15,16)$. The TRAF6 protein is composed of four domains; a coiled-coil structural domain (also known as TRAF-N structural domain) and a conservative TRAF-C structural domain are contained in its C-terminal, which can interact with upstream receptors or other signaling proteins through self-association, thus resulting in exertion of its biological function $(38,39)$. The N-terminal of the TRAF6 protein contains a RING finger structural domain (E3 ubiquitin ligase) and multiple zinc finger domains (bound with specific DNA sequences); these structures have important roles in the activation of downstream signals (40). In recent years, studies have revealed that TRAF6 has an important role in the development, invasion and metastasis of tumors by activating $\mathrm{NF}-\kappa \mathrm{B}(41,42)$. Our preliminary results indicated that TRAF6 expression is increased in osteosarcoma tissues; therefore it may be considered a proto-oncogene. These findings were validated in the present study. The present study aimed to explore the possible mechanism underlying the regulation of TRAF6 in osteosarcoma.

miRNAs are a class of evolutionarily conserved, non-protein coding RNAs (43-45), which are widely expressed in numerous organisms. Through the transcriptional regulation of target genes, miRNAs are involved in the regulation of several types of tumorigenesis and metastasis $(46,47)$. In the present study, the miRNA that may regulate TRAF6 expression was analyzed using a series of online software, thus resulting in the identification of miR-124. Existing research indicated that through the downregulation of Rho-associated protein kinase 1, miR-124 may inhibit the proliferation and migration of colon cancer cells (48). In astrocytoma, miR-124 has been reported to regulate cell proliferation, apoptosis and migration via proto-oncogene serine/threonine-protein kinase Pim-1 (49). In the present study, the results of a luciferase reporter gene system demonstrated that in the miR-124 mimic group, luciferase activity was significantly decreased, and western blotting revealed that the overexpression of miR-124 inhibited the expression of TRAF6. These findings verified TRAF6 as a target gene regulated by miR-124.

In the present study, RT-qPCR was used to detect the expression levels of miR-124 in osteosarcoma tissues; the results revealed that $\mathrm{miR}-124$ expression was downregulated in osteosarcoma tissues and cells. Subsequently, miR-124 was overexpressed in MG-63 cells via transfection with a miR-124 mimic. The biological effects of miR-124 overexpression were then examined using MTT, flow cytometry and Transwell invasion assay analytical methods. The results demonstrated that miR-124 overexpression decreased cell viability, inhibited cell cycle progression and invasion, and promoted apoptosis. 
Therefore, it may be hypothesized that miR-124 participates in the occurrence and metastasis of the osteosarcoma malignant phenotype by regulating TRAF6 and associated downstream signaling pathways. In the present study, miR-124 overexpression in osteosarcoma cells reduced TRAF6 expression, thus inhibiting cell cycle progression and invasion of osteosarcoma cells, and promoting osteosarcoma cell apoptosis.

In order to further verify the role of TRAF6 in cell biology via miR-124 regulation, the present study constructed a TRAF6 recombinant expression vector, pcDNA3.1-TRAF6. Subsequently, MG-63 cells were cotransfected with pcDNA3.1-TRAF6 and a miR-124 mimic, and MTT, flow cytometry and Transwell invasion assay analytical methods were used to analyze the effects. The results suggested that miR-124 overexpression could reverse the effects of TRAF6 overexpression on osteosarcoma cells, and promote apoptosis.

In conclusion, downregulation of miR-124 in human osteosarcoma tissues and cell lines may increase the expression of TRAF6 protein. miR-124 may inhibit cell cycle progression and invasion of osteosarcoma cells, and promote apoptosis by regulating TRAF6. Therefore, therapeutic strategies that enhance the expression of miR-124 or inhibit the expression of TRAF6 may benefit patients with osteosarcoma.

\section{Acknowledgements}

Not applicable.

\section{Funding}

The present study was supported by the National Natural Science Foundation of China (grant no. 81402226).

\section{Availability of data and materials}

All data generated or analyzed during this study are included in this published article.

\section{Authors' contributions}

$\mathrm{QM}$ and WZ designed the research and wrote the manuscript. XX, JL, HM, XL, LQ, XZ and MZ collected the data, analyzed and interpreted the data. All authors read and approved the final manuscript.

\section{Ethics approval and consent to participate}

The present study was approved by the Ethics Committee of Yancheng City No. 1 People's Hospital (Yancheng, China; approval no. 2015YCYY-0001). The patients provided written informed consent prior to sample collection.

\section{Consent for publication}

The patients provided written informed consent for the use of data in this study.

\section{Competing interests}

The authors declare that they have no competing interests.

\section{References}

1. Lugowska I, Mierzejewska E, Lenarcik M, Klepacka T, Koch I, Michalak E, Szamotulska K: The clinical significance of changes in ezrin expression in osteosarcoma of children and young adults. Tumour Biol 37: 12071-12078, 2016.

2. Lee JA, Jeon DG, Cho WH, Song WS, Yoon HS, Park HJ, Park BK, Choi HS, Ahn HS, Lee JW, et al: Higher gemcitabine dose was associated with better outcome of osteosarcoma patients receiving gemcitabine-docetaxel chemotherapy. Pediatr Blood Cancer 63: 1552-1556, 2016.

3. Jiang H, Liu W, Li G, Fan T and Mao B: Chinese medicinal herbs in the treatment of upper airway cough syndrome: A systematic review of randomized, controlled trials. Altern Ther Health Med 22: 38-51, 2016.

4. Wang L, Yang L, Lu Y, Chen Y, Liu T, Peng Y, Zhou Y, Cao Y, Bi Z, Liu T, et al: Osthole induces cell cycle arrest and inhibits migration and invasion via PTEN/Akt pathways in osteosarcoma. Cell Physiol Biochem 38: 2173-2182, 2016.

5. Ali Gumustas S, Isyar M, Topuk S, Yilmaz I, Oznam K, Onay T, Ofluoglu O and Mahirogullari M: Systematic evaluation of drug-loaded hydrogels for application in osteosarcoma treatment. Curr Pharm Biotechnol 17: 866-872, 2016.

6. Kebudi R, Ozger H, Kızılocak H, Bay SB and Bilgiç B: Osteosarcoma after hematopoietic stem cell transplantation in children and adolescents: Case Report and Review of the Literature. Pediatr Blood Cancer 63: 1664-1666, 2016.

7. Cheng L, Wang C and Jing J: Cell cycle kinases in osteosarcoma: Potential for therapeutic intervention. Curr Pharm Des 22: 4830-4834, 2016.

8. Maximov VV and Aqeilan RI: Genetic factors conferring metastasis in osteosarcoma. Future Oncol 12: 1623-1644, 2016.

9. Li X, Zhang Y, Wan S, Li H, Li D, Xia J, Yuan Z, Ren M, Yu S, Li S et al: A comparative study between limb-salvage and amputation for treating osteosarcoma. J Bone Oncol 5: 15-21, 2016.

10. Wan J, Zhang X, Liu T and Zhang X: Strategies and developments of immunotherapies in osteosarcoma. Oncol Lett 11: 511-520, 2016

11. Durham BH, Diamond EL and Abdel-Wahab O: Histiocytic neoplasms in the era of personalized genomic medicine. Curr Opin Hematol 23: 416-425, 2016.

12. Cagle PT, Raparia K and Portier BP: Emerging biomarkers in personalized therapy of lung cancer. Adv Exp Med Biol 890: 25-36, 2016.

13. Krishna A, Singh S, Kumar V and Pal US: Molecular concept in human oral cancer. Natl J Maxillofac Surg 6: 9-15, 2015.

14. De P, Carlson JH, Leyland-Jones B and Dey N: Role of 'oncogenic nexus' of CIP2A in breast oncogenesis: How does it work? Am J Cancer Res 5: 2872-2891, 2015.

15. Luo Z, Zhang X, Zeng W, Su J, Yang K, Lu L, Lim CB, Tang W, Wu L, Zhao S, et al: TRAF6 regulates melanoma invasion and metastasis through ubiquitination of Basigin. Oncotarget 7: 7179-7192, 2016

16. Han F, Zhang L, Qiu W and Yi X: TRAF6 promotes the invasion and metastasis and predicts a poor prognosis in gastric cancer. Pathol Res Pract 212: 31-37, 2016.

17. Lin FT, Lin VY, Lin VT and Lin WC: TRIP6 antagonizes the recruitment of A20 and CYLD to TRAF6 to promote the LPA2 receptor-mediated TRAF6 activation. Cell Discov 2: 15048, 2016.

18. Zhao T, Tang X, Umeshappa CS, Ma H, Gao H, Deng Y, Freywald A and Xiang J: Simulated microgravity promotes cell apoptosis through suppressing Uev1A/TICAM/TRAF/NF- $\kappa \mathrm{B}$-regulated anti-apoptosis and p53/PCNA- and ATM/ATR-Chk1/2-controlled DNA-damage response pathways. J Cell Biochem 117: 2138-2148, 2016.

19. Panda S, Nilsson JA and Gekara NO: Deubiquitinase MYSM1 regulates innate immunity through inactivation of TRAF3 and TRAF6 complexes. Immunity 43: 647-659, 2015.

20. Tomomura M, Suzuki R, Shirataki Y, Sakagami H, Tamura N, Tomomura A and Rhinacanthin C: Rhinacanthin C inhibits osteoclast differentiation and bone resorption: Roles of TRAF6/TAK1/MAPKs/NF-кB/NFATc1 signaling. PLoS One 10: $\mathrm{e} 0130174,2015$.

21. Kong X, Yang Y, Wu W, Wan H, Li X, Zhong M, Su X, Jia S and Lin $\mathrm{N}$ : Triterpenoid saponin W3 from Anemone flaccida suppresses osteoclast differentiation through inhibiting activation of MAPKs and NF- $\kappa B$ pathways. Int J Biol Sci 11: 1204-1214, 2015.

22. Kong X, Wu W, Yang Y, Wan H, Li X, Zhong M, Zhao H, Su X, Jia S, Ju D, et al: Total saponin from Anemone flaccida $\mathrm{Fr}$. Schmidt abrogates osteoclast differentiation and bone resorption via the inhibition of RANKL-induced NF- $\kappa B$, JNK and p38 MAPKs activation. J Transl Med 13: 91, 2015. 
23. He Z, Huang C, Lin G and Ye Y: siRNA-induced TRAF6 knockdown promotes the apoptosis and inhibits the invasion of human lung cancer SPC-A1 cells. Oncol Rep 35: 1933-1940, 2016.

24. Peng Z, Shuangzhu Y, Yongjie J, Xinjun Z and Ying L: Retraction note to: TNF receptor-associated factor 6 regulates proliferation, apoptosis, and invasion of glioma cells. Mol Cell Biochem 415 207, 2016.

25. Han Q, Yao F, Zhong C and Zhao H: TRAF6 promoted the metastasis of esophageal squamous cell carcinoma. Tumour Biol 35: 715-721, 2014.

26. Sun H, Li XB, Meng Y, Fan L, Li M and Fang J: TRAF6 upregulates expression of HIF-1 $\alpha$ and promotes tumor angiogenesis. Cancer Res 73: 4950-4959, 2013.

27. Meng Q, Zheng M, Liu H, Song C, Zhang W, Yan J, Qin L and Liu X: TRAF6 regulates proliferation, apoptosis, and invasion of osteosarcoma cell. Mol Cell Biochem 371: 177-186, 2012.

28. Peng S, Gao D, Gao C, Wei P, Niu M and Shuai C: MicroRNAs regulate signaling pathways in osteogenic differentiation of mesenchymal stem cells (Review). Mol Med Rep 14: 623-629, 2016.

29. Yeh CH,Moles R and NicotC: Clinical significance of microRNAs in chronic and acute human leukemia. Mol Cancer 15: 37, 2016.

30. Feng T, Shao F, Wu Q, Zhang X, Xu D, Qian K, Xie Y, Wang S, $\mathrm{Xu}$ N, Wang Y, et al: miR-124 downregulation leads to breast cancer progression via LncRNA-MALAT1 regulation and CDK4/E2F1 signal activation. Oncotarget 7: 16205-16216, 2016.

31. Zhang H, Wang Q, Zhao Q and Di W: MiR-124 inhibits the migration and invasion of ovarian cancer cells by targeting SphK1. J Ovarian Res 6: 84, 2013.

32. Denduluri SK, Wang Z, Yan Z, Wang J, Wei Q, Mohammed MK, Haydon RC, Luu HH and He TC: Molecular pathogenesis and therapeutic strategies of human osteosarcoma. J Biomed Res: Aug 30, 2015 (Epub ahead of print).

33. Gu X, Ding J, Zhang Z, Li Q, Zhuang X and Chen X: Polymeric nanocarriers for drugdelivery in osteosarcoma treatment. Curr Pharm Des 21: 5187-5197, 2015.

34. Sampson VB, Yoo S, Kumar A, Vetter NS and Kolb EA: MicroRNAs and potential targets in osteosarcoma: Review. Front Pediatr 3: 69, 2015.

35. Lim HJ and Yang JL: Regulatory roles and therapeutic potential of microRNA in sarcoma. Crit Rev Oncol Hematol 97: 118-130, 2016.

36. Morrow JJ and Khanna C: Osteosarcoma genetics and epigenetics: Emerging biology and candidate therapies. Crit Rev Oncog 20: 173-197, 2015.

37. Isakoff MS, Bielack SS, Meltzer P and Gorlick R: Osteosarcoma: Current treatment and a collaborative pathway to success. J Clin Oncol 33: 3029-3035, 2015.
38. Liu H, Tamashiro S, Baritaki S, Penichet M, Yu Y, Chen H, Berenson $\mathrm{J}$ and Bonavida B: TRAF6 activation in multiple myeloma: A potential therapeutic target. Clin Lymphoma Myeloma Leuk 12: 155-163, 2012.

39. Kumar A, Bhatnagar S and Paul PK: TWEAK and TRAF6 regulate skeletal muscle atrophy. Curr Opin Clin Nutr Metab Care 15: 233-239, 2012.

40. Landström M: The TAK1-TRAF6 signalling pathway. Int J Biochem Cell Biol 42: 585-589, 2010.

41. Yan J, Yin Y, Zhong W, Wang C and Wang Z: CD137 Regulates NFATc1 expression in mouse VSMCs through TRAF6/NF-kappaB p65 signaling pathway. Mediators Inflamm 2015: 639780, 2015

42. Shi Z, Zhang Z, Zhang Z, Wang Y, Li C, Wang X, He F, Sun L, Jiao S, Shi W, et al: Structural Insights into mitochondrial antiviral signaling protein (MAVS)-tumor necrosis factor receptor-associated factor 6 (TRAF6) signaling. J Biol Chem 290: 26811-26820, 2015.

43. Tian X and Zhang X: A single nucleotide polymorphism (rs1056629) in 3'-UTR of MMP-9 is responsible for a decreased risk of metastatic osteosarcoma by compromising its interaction with microRNA-491-5p. Cell Physiol Biochem 38: 1415-1424, 2016.

44. Zhu Z, Tang J, Wang J, Duan G, Zhou L and Zhou X: MiR-138 acts as a tumor suppressor by targeting EZH2 and enhances cisplatin-induced apoptosis in osteosarcoma cells. PLoS One 11: e0150026, 2016.

45. Wu S, Du X, Wu M, Du H, Shi X and Zhang T: MicroRNA-409-3p inhibits osteosarcoma cell migration and invasion by targeting catenin- $\delta 1$. Gene 584: 83-89, 2016

46. Bhattacharya S, Chalk AM, Ng AJ, Martin TJ, Zannettino AC, Purton LE, Lu J,BakerEK and Walkley CR: Increased miR-155-5p and reduced miR-148a-3p contribute to the suppression of osteosarcoma cell death. Oncogene 35: 5282-5294, 2016.

47. Pu Y, Zhao F, Cai W, Meng X, Li Y and Cai S: MiR-193a-3p and miR-193a-5p suppress the metastasis of human osteosarcoma cells by downregulating Rab27B and SRR, respectively. Clin Exp Metastasis 33: 359-372, 2016.

48. Zhou L, Xu Z, Ren X, Chen K and Xin S: MicroRNA-124 (MiR-124) inhibits cell proliferation, metastasis and invasion in colorectal cancer by downregulating Rho-associated protein kinase 1 (ROCK1). Cell Physiol Biochem 38: 1785-1795, 2016.

49. Deng D, Wang L, Chen Y, Li B, Xue L, Shao N, Wang Q, Xia X, Yang Y and Zhi F: miR-124-3p regulates cell proliferation, invasion, apoptosis and bioenergetics by targeting PIM1 in astrocytoma. Cancer Sci 107: 899-907, 2016. 